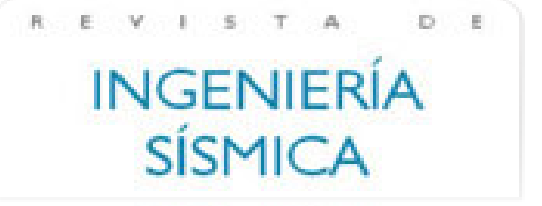

Revista de Ingeniería Sísmica

ISSN: 0185-092X

javiles@tlaloc.imta.mx

Sociedad Mexicana de Ingeniería Sísmica

México

Chan Dorado, Selma M.; Ruiz Gómez, Sonia E.; Montiel Ortega, Marco A.

Escalamiento de acelerogramas y número mínimo de registros requeridos para el análisis de estructuras

Revista de Ingeniería Sísmica, núm. 72, enero-junio, 2005, pp. 1-24

Sociedad Mexicana de Ingeniería Sísmica

Distrito Federal, México

Disponible en: http://www.redalyc.org/articulo.oa?id=61807202

Cómo citar el artículo

- Número completo

- Más información del artículo

Página de la revista en redalyc.org

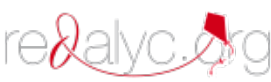

Sistema de Información Científica

Red de Revistas Científicas de América Latina, el Caribe, España y Portugal

Proyecto académico sin fines de lucro, desarrollado bajo la iniciativa de acceso abierto 


\title{
ESCALAMIENTO DE ACELEROGRAMAS Y NÚMERO MÍNIMO DE REGISTROS REQUERIDOS PARA EL ANÁLISIS DE ESTRUCTURAS
}

\author{
Selma M. Chan Dorado ${ }^{(1)}$, Sonia E. Ruiz Gómez ${ }^{(2)}$ y Marco A. Montiel Ortega ${ }^{(2)}$
}

\begin{abstract}
RESUMEN
Se explora el uso de diferentes criterios de escalamiento de movimientos sísmicos de banda estrecha. Estos corresponden a catorce acelerogramas registrados en suelo blando de la Ciudad de México, específicamente en el patio de la Secretaría de Comunicaciones y Transportes (estación SCT). Los movimientos sísmicos se utilizan para evaluar la confiabilidad estructural de tres estructuras de concreto reforzado (de 5, 10 y 15 pisos, y tres crujías) diseñadas de acuerdo con el Reglamento de Construcciones del Distrito Federal. Se estima el mínimo número de movimientos sísmicos (asociados a una banda de confianza) que deben utilizarse para el análisis. Dicho número mínimo de movimientos sísmicos depende de la dispersión de las distorsiones máximas de entrepiso producidas por movimientos sísmicos con un periodo de recurrencia dado.
\end{abstract}

\begin{abstract}
The use of different seismic motion scaling criteria is explored through several narrow-band accelerograms. These correspond to fourteen records obtained on soft soil in Mexico City, specifically in the Ministry of Communications and Transportation site (SCT site). The motions are used for the reliability evaluation of three structures (5-, 10-and 20-story, 3-bay frames) designed in accordance with the Mexico City Seismic Code (RCDF). The minimum number of seismic motions (associated with a confidence band) that should be used in the analysis is estimated. This number of motions depends on the dispersion of the maximum story drifts produced by seismic motions with a given return period.
\end{abstract}

\section{INTRODUCCIÓN}

La respuesta sísmica probabilista de estructuras con comportamiento no lineal sujetas a sismos entre moderados y muy intensos puede estimarse usando diferentes métodos de análisis, entre ellos el método dinámico paso a paso en el tiempo. En este caso, las estructuras se someten a la acción de un conjunto de movimientos sísmicos asociados con ciertos periodos de recurrencia, y

Artículo recibido el 26 de marzo de 2003 y aprobado para su publicación el 28 de septiembre de 2004. Se aceptarán comentarios y/o discusiones hasta cinco meses después de su publicación.

(1) Universidad Politécnica de Cataluña, c/Jordi Girona 1-3, Módulo D-2, 08034, Barcelona, España, antes en el Instituto de Ingeniería, UNAM. selma.chan@upc.edu.

(2) Instituto de Ingeniería, UNAM, Ciudad Universitaria, 04510 México, DF. SRuizG@iingen.unam.mx; mmo@pumas.iingen.unam.mx. 
a partir de estos análisis se obtienen los parámetros estadísticos de la respuesta que interesa ( $\mathrm{p}$. ej.: distorsiones máximas de entrepiso, desplazamiento máximo de azotea, ductilidades máximas globales o locales, energía disipada normalizada, etc.). Los parámetros estadísticos que normalmente interesa conocer son la mediana y la desviación estándar de la respuesta. Por ejemplo, el estimador que interesa en este estudio es la mediana de las distorsiones máximas de entrepiso.

De lo anterior, surge la pregunta ¿cuántos acelerogramas se deben utilizar en el análisis de manera que el estimador seleccionado tenga cierto intervalo de confianza? Otras preguntas son ¿cómo seleccionar dichos movimientos? y ¿cómo escalarlos?

Las respuestas a estas preguntas han sido discutidas por varios autores (Matsumura, 1992; Shome et al., 1998; Shome y Cornell, 1999) quienes han sentado bases para el estudio de este tema. Las conclusiones a las que ha llegado del grupo del Prof. Cornell se basan en la respuesta probabilista de marcos de acero resistentes a flexión, sujetos a movimientos sísmicos de banda ancha registrados en terreno duro en California, EUA.

En el presente artículo se analizan marcos de concreto reforzado resistentes a flexión, diseñados de acuerdo con el Reglamento de Construcciones del Distrito Federal (1976 y 1993), sujetos a movimientos de banda estrecha registrados en terreno blando, específicamente en el patio de la Secretaría de Comunicaciones y Transportes (SCT) en la Ciudad de México.

En este estudio se analiza la influencia que tienen distintos criterios de escalamiento en el número mínimo de movimientos sísmicos que se requieren para garantizar que el estimador (que en este trabajo es la mediana de la distorsión máxima de entrepiso) tenga un intervalo de confianza dado. Cuanto más pequeño es el número de acelerogramas requerido, más ahorro se tiene en tiempo de procesamiento de datos en computadora, pero por otro lado también es menor la banda de confianza del estimador.

Se analizan tres marcos estructurales de concreto reforzado sujetos a diferentes intensidades sísmicas. Se identifica el método de escalamiento que da lugar al menor número de registros sísmicos requeridos para que el estimador tenga cierto nivel de confianza. Además, para cada estructura se obtiene la curva de peligro de demanda estructural y su correspondiente función de probabilidad de excedencia de la distorsión máxima de entrepiso para un intervalo de 50 años. Las probabilidades de excedencia que se calculan en este estudio proporcionan idea sobre la confiabilidad implícita en las construcciones diseñadas de acuerdo con el RCDF, localizadas en el sitio SCT de la ciudad de México.

\section{FACTOR DE ESCALA}

Para excitar las estructuras con diferentes niveles de intensidad sísmica es necesario modificar las amplitudes de los movimientos sísmicos registrados por un factor de escala. Este es un valor numérico que multiplica a la historia de aceleraciones del registro, de tal manera que la ordenada espectral correspondiente al periodo fundamental de la estructura por analizar tenga una 
intensidad especificada. Dicha intensidad se asocia a cierto periodo de retorno. Este se obtiene a partir de una curva de peligro sísmico, según el nivel de desempeño que se trate.

El procedimiento para calcular el factor de escala es como sigue:

a) Se obtiene el periodo fundamental de vibrar $\left(T_{\mathrm{o}}\right)$ de la estructura en estudio.

b) Se proponen los periodos de retorno de los sismos $\left(T_{R}\right)$ para los cuales se revisará la estructura.

c) Con el periodo de retorno propuesto en el paso (b) se encuentra la intensidad correspondiente.

d) Se calcula el valor del espectro de resistencia por unidad de masa de cada registro en estudio, correspondiente al periodo $T_{o}$, para la misma demanda de ductilidad y amortiguamiento crítico considerado en la curva de peligro sísmico.

e) El factor de escala correspondiente al registro en cuestión se calcula como el cociente de la intensidad obtenida de la curva de peligro sísmico, y la obtenida a partir del espectro de respuesta.

Para este estudio se seleccionaron los registros sísmicos que aparecen en la tabla 1 , correspondientes a la estación ubicada en el patio de la Secretaría de Comunicaciones y Transportes (SCT) en la ciudad de México. Todos los eventos sísmicos a los que corresponden estos movimientos son de subducción, con magnitud mayor que o igual a 5.6. Los acelerogramas fueron previamente cortados y filtrados para corregir su línea base (Villa y Ruiz, 2001).

Tabla 1. Características de los registros sísmicos

\begin{tabular}{|c|c|c|c|c|c|c|}
\hline \multirow{2}{*}{\begin{tabular}{|c|} 
Número \\
de \\
sismo
\end{tabular}} & \multirow[b]{2}{*}{ Fecha } & \multirow{2}{*}{\begin{tabular}{|c|} 
Magnitud \\
(M) \\
\end{tabular}} & \multirow{2}{*}{$\begin{array}{l}\text { Distancia } \\
\text { epicentral } \\
(\mathbf{k m})\end{array}$} & \multirow{2}{*}{\begin{tabular}{|c|} 
Periodo \\
dominante \\
$(\mathrm{s})$
\end{tabular}} & \multicolumn{2}{|c|}{ Coordenadas del epicentro } \\
\hline & & & & & Latitud N & Long.W \\
\hline 1 & 19-sep-85 EW & 8.1 & 441.69 & 2.03 & 18.081 & 102.942 \\
\hline 2 & 19-sep-85 NS & 8.1 & 441.69 & 2.05 & 18.081 & 102.942 \\
\hline 3 & 25-abr-89 EW & 6.9 & 308.16 & 2.07 & 16.603 & 99.400 \\
\hline 4 & 25 -abr-89 NS & 6.9 & 308.16 & 2.19 & 16.603 & 99.400 \\
\hline 5 & 24-oct-93 EW & 6.6 & 314.37 & 1.83 & 16.540 & 98.980 \\
\hline 6 & 24-oct-93 NS & 6.6 & 314.37 & 1.73 & 16.540 & 98.980 \\
\hline 7 & 10-dic-94 EW & 6.3 & 305.39 & 1.87 & 18.020 & 101.560 \\
\hline 8 & 10 -dic-94 NS & 6.3 & 305.39 & 2.02 & 18.020 & 101.560 \\
\hline 9 & 22-may-97 EW & 6.0 & 312.25 & 1.90 & 18.410 & 101.810 \\
\hline 10 & 22-may-97 NS & 6.0 & 312.25 & 1.50 & 18.410 & 101.810 \\
\hline 11 & 31-may-90 EW & 5.9 & 316.60 & 1.98 & 17.106 & 100.893 \\
\hline 12 & 31-may-90 NS & 5.9 & 316.60 & 1.50 & 17.106 & 100.893 \\
\hline 13 & 23-may-94 EW & 5.6 & 216.75 & 1.60 & 18.030 & 100.570 \\
\hline 14 & 23-may-94 NS & 5.6 & 216.75 & 1.65 & 18.030 & 100.570 \\
\hline
\end{tabular}


En la fig. 1 se muestran los espectros de seudoaceleración de los 14 movimientos sísmicos escalados para un periodo de retorno de 50 años y periodo fundamental de vibración $T_{o}=1 \mathrm{~s}$. En la tabla 1 y la fig. 1 se puede ver que los espectros correspondientes a eventos con magnitudes (M) iguales a o menores que 6 (sismos 9 a 14) en general presentan periodos dominantes menores que los de mayor magnitud (sismos 1 a 8). Con el fin de observar esto con mayor claridad en la fig. 1 se presentan (con línea negra gruesa) los promedios correspondientes a los espectros de estos dos grupos de sismos. En la fig. se puede observar que los sismos con magnitudes pequeñas dan lugar a movimientos que tienen formas espectrales que son diferentes a los de magnitudes grandes. En este estudio se seleccionaron solamente movimientos correspondientes a sismos con magnitudes grandes (mayores que 5.6), y con periodos dominantes entre 1.5 y $2.2 \mathrm{~s}$ (fig. 1). Estos movimientos no son representativos de sismos con intensidades pequeñas; sin embargo, los errores que se cometen al estimar el número de sismos requeridos para el análisis de estructuras ante movimientos con periodos de recurrencia pequeños (p. ej. 10 años) son poco significativos.

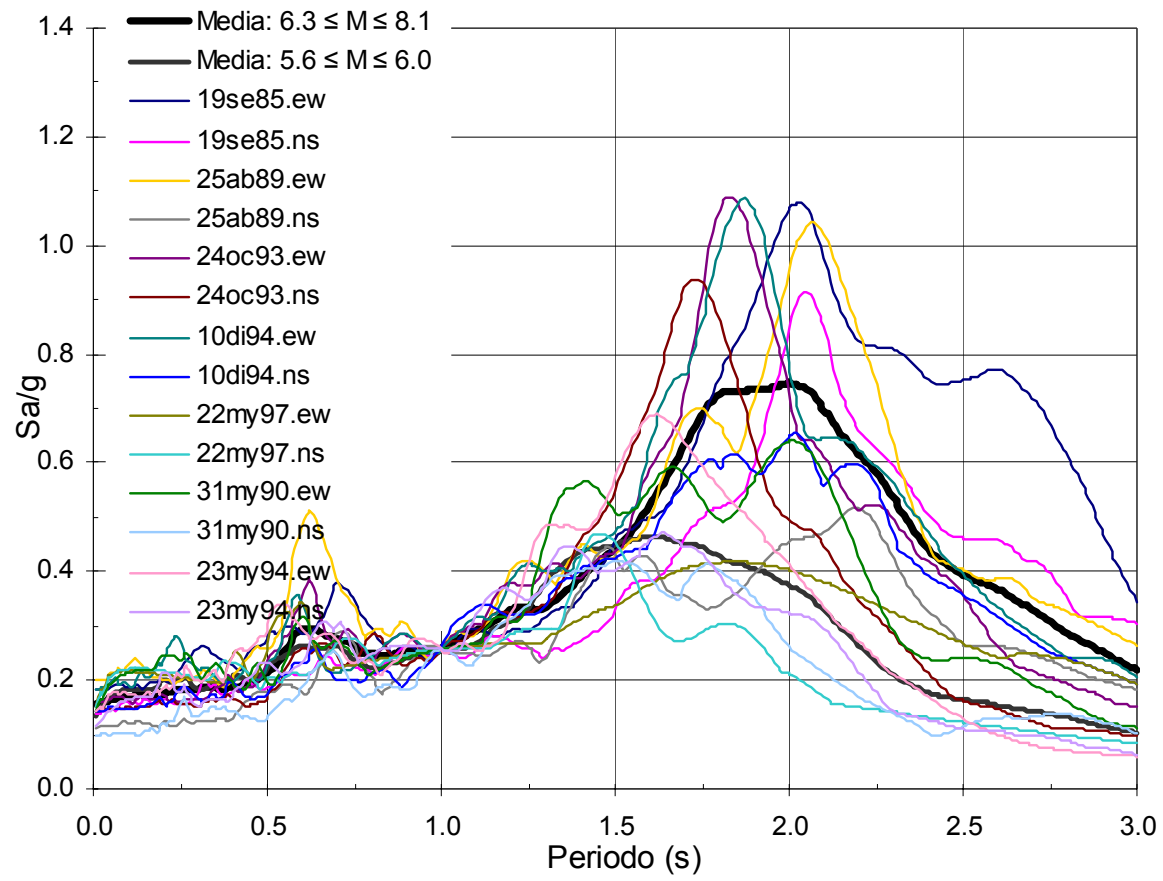

Figura 1. Espectros de seudoaceleración de 14 movimientos registrados en la estación SCT

\section{CRITERIOS DE ESCALAMIENTO DE ACELEROGRAMAS}

Los criterios de escalamiento de acelerogramas se relacionan con el comportamiento que presentan las estructuras ante la acción sísmica. Las características que se toman en cuenta son: la demanda de ductilidad ( $\mu$ ), el amortiguamiento $(\xi)$, y el periodo fundamental de vibrar de las estructura en estudio $\left(T_{o}\right)$. Los criterios de escalamiento que se consideran en este estudio son los siguientes 
a) $\mu=1, \xi=5 \%$, y periodo fundamental de vibrar $T_{\mathrm{o}}$

b) $\mu=1, \xi=20 \%, y T_{\text {o }}$

c) $\mu=3, \xi=5 \%, y T_{\mathrm{o}}$

d) $\mu=1, \xi=20 \%$, y factores de peso modal

En el primer criterio de escalamiento (inciso a) se utilizan curvas y espectros de respuesta lineal elástica $(\mu=1)$, correspondientes a sistemas con amortiguamiento crítico $\xi=5 \%$ y periodo fundamental de vibrar $T_{\mathrm{o}}$. Comúnmente los factores de escala se obtienen según este criterio, debido a que las curvas de peligro sísmico que se calculan normalmente corresponden a los valores de $\mu$ y $\xi$ antes mencionados.

Una característica del comportamiento inelástico es que el porcentaje de amortiguamiento crítico "equivalente" que originalmente tiene el edificio, aumenta a medida que la acción sísmica es más grande. Es por eso que se analiza el criterio del inciso $(b)$.

En el criterio del inciso $(c)$ se supone una ductilidad $\mu=3$. Este valor se asocia al factor de comportamiento sísmico de diseño. El criterio del inciso $(d)$ considera el hecho que los modos superiores de vibrar sean significativos en la respuesta, por lo que se utilizan factores de peso que dependen de la masa modal efectiva de cada modo.

En todos los casos se emplea el método de análisis dinámico paso a paso; sin embargo, el criterio con el que se escalan los acelerogramas es diferente en cada caso (incisos $a-d$ ).

\section{MÉTODO SIMPLIFICADO PARA EVALUAR LA CONFIABILIDAD}

En lo que sigue se describe de manera general el método utilizado en este estudio para evaluar la confiabilidad de las estructuras. Se trata del método simplificado propuesto por Cornell (1996). integral:

La curva de peligro de la demanda estructural puede obtenerse mediante la siguiente

$$
v_{D}(d)=\int-\frac{\partial v(y)}{\partial y} P(D \geq d \mid y) \cdot d y
$$

donde $v(y)$ es la tasa media anual de excedencia de la intensidad estudiada. $P(D \geq d \mid y)$ es la probabilidad condicional de que la distorsión máxima de entrepiso $D$ exceda cierto nivel $d$, debido a la acción de un sismo de intensidad $y$. En este caso la intensidad $y$ se toma como la seudoaceleración normalizada respecto a la gravedad, $\mathrm{Sa} / \mathrm{g}$.

Si se supone que la función de distribución de probabilidad de la distorsión máxima de entrepiso es lognormal, se llega a (ver deducción en el Apéndice A): 


$$
P(D \geq d \mid y)=1-\Phi\left(\frac{\ln (d / \hat{D}}{\sigma_{\ln D}}\right)
$$

donde $\hat{D}$ es la mediana de $D$ y $\sigma_{\ln D}$ la desviación estándar de su logaritmo natural. Como se indica más adelante, $\hat{D}$ y $\sigma_{\ln D}$ son funciones de $S_{a} / g$.

Con el fin de llegar a una ecuación simplificada en lugar de la integral de la ec. 1, Cornell (1996) supone que la tasa media anual de excedencia $v$, y que la variación de la mediana $(\hat{D})$ con la intensidad, pueden expresarse mediante:

$$
\begin{aligned}
& v=k(S a / g)^{-r} \\
& \hat{D}=a(S a / g)^{b}
\end{aligned}
$$

donde $k, r$ son los parámetros de regresión de la curva que representa la tasa media anual de excedencia de $\mathrm{Sa} / \mathrm{g}$; y $a$ y $b$ son los parámetros de ajuste de la mediana de la máxima distorsión de entrepiso. Sustituyendo las ecs. 3 y 4 en la ec. 1, Cornell (1996) llega a la siguiente expresión para obtener la curva de peligro de la demanda estructural, $v_{D}(d)$ :

$v_{D}(d)=k\left[S_{a} / g\right]^{-r} \exp \left[\frac{1}{2}\left(\frac{r}{b} \sigma_{\ln D}\right)^{2}\right]$

Esta puede expresarse como sigue:

$$
v_{D}(d)=v F_{c}
$$

donde $v$ representa la curva de peligro sísmico, y $F_{c}$ se puede interpretar como un "factor de corrección" dado por:

$$
F_{c}=\exp \left[\frac{1}{2}\left(\frac{r}{b} \sigma_{\ln D}\right)^{2}\right]
$$

Los autores del presente estudio compararon los resultados del método exacto (ec. 1) con los del simplificado (ec. 5) correspondientes a edificios de cinco y de diez niveles (Montiel et al., 2002; Montiel et al., 2003), y concluyeron que para esas estructuras ambos métodos dan lugar a confiabilidades similares. Con base en esa comparación, aquí utilizamos el método simplificado de análisis. 


\section{NÚMERO MÍNIMO DE ACELEROGRAMAS}

En la introducción de este artículo se planteó la pregunta ¿cuántos acelerogramas se deben utilizar en el análisis de manera que el estimador seleccionado tenga cierto intervalo de confianza? En este caso es necesario definir un intervalo de confianza debido a que se está trabajando con un número limitado de acelerogramas. Cuanto más grande sea el número de acelerogramas, el intervalo de confianza en mayor, pero el tiempo de cómputo requerido para el análisis dinámico también es mayor.

En la sección anterior se mencionó que se considera que la distorsión máxima de entrepiso $D$ tiene fdp lognormal con mediana $\hat{D}$ (el estimador seleccionado), por tanto el logaritmo natural de D tiene fdp normal con media $\ln \hat{D}$ (Benjamin y Cornell, 1970). Debido a esto se pueden usar resultados a partir de una fdp normal (se obtiene un intervalo de confianza sobre $\ln \hat{D})$.

Con el fin de encontrar el número de acelerogramas $(n)$ que se requieren para que el estimador $(\hat{D})$ tenga cierto nivel de confianza es necesario conocer la desviación estándar de ln $D$, así como proponer un valor del error $(e)$ que se desea admitir al usar $\hat{D}$ para estimar el valor de la mediana de la población. En este artículo se utiliza la siguiente expresión para encontrar el valor de $n$. Las hipótesis que hay detrás de esta expresión, así como su deducción, se presentan en el Apéndice $\mathrm{B}^{(1)}$ :

$$
n=K_{\alpha / 2}^{2} \frac{\sigma_{\ln D}^{2}}{e^{2}}
$$

donde $\left(K_{\alpha / 2}\right)$ está directamente relacionado con el nivel de confianza $(1-\alpha)$ deseado (Apéndice $\left.\mathrm{B}^{(1)}\right)$. Si se desea obtener un estimador (mediana de las distorsiones máximas) con un valor $e=$ $\pm 10 \%$ con $95 \%$ de confianza (eso implica que $K_{\alpha / 2}=2$ ) se deberá usar un número de registros igual a $n=4 \sigma_{\ln D}^{2} / 0.10^{2}$; sin embargo, si se desea un porcentaje de confianza menor, de por ejemplo $68 \%$, entonces $K_{\alpha / 2}=1$, y la ec. 8 se convierte en:

$$
n=\frac{\sigma_{\ln D}^{2}}{0.010}
$$

Las desviaciones estándar promedio del logaritmo de las distorsiones máximas $\sigma_{\ln D}$, para periodos de retorno de 125 años, tienen valores aproximadamente entre 0.17 y 0.3 . Si se sustituyen estos valores en la ec. 9 se encuentra que el valor entero de $n$ resulta entre 3 y 9 .

(1) Se hace la aclaración que las fórmulas del Apéndice B y, por tanto, la fórmula para determinar el tamaño de la muestra (ec. 8) corresponden a la conocida teoría de muestreo en poblaciones con distribución normal; pero aquí se le asigna a la distorsión máxima de entrepiso, $D$, una fdp lognormal. Por tanto, el intervalo de confianza no se está obteniendo sobre la mediana, sino sobre el logaritmo de la mediana, que es igual a la media de la distribución (normal) de $\ln D$. 
Los reglamentos de diseño generalmente recomiendan que se utilicen entre $n=3$ y 7 registros para el análisis dinámico de las estructuras (dependiendo del reglamento de diseño que se trate). Es decir, que si se toman como base los valores que se utilizan en la práctica $(3 \leq n \leq 7)$, entonces resulta adecuado que el estimador se calcule con una confianza de 68\% (esto implica $K_{\alpha / 2}=1.0$ ), y también que se acepte un valor de $e=10 \%$. Debido a lo anterior, los autores decidieron emplear en este estudio los valores implícitos en la ec. 9; sin embargo, no hay una base para afirmar que estos valores son los óptimos, por lo que sería deseable realizar un análisis de optimación de estos parámetros y su implicación en el análisis de confiabilidad de las estructuras.

\section{PROCEDIMIENTO DE ANÁLISIS}

En los ejemplos que se presentan más adelante se aplica el siguiente procedimiento de análisis:

Con la curva de peligro sísmico y las ordenadas espectrales asociadas al periodo fundamental de la estructura (correspondientes al criterio de escalamiento considerado) se calculan los factores de escala.

Los registros sísmicos se afectan por los factores de escala para realizar el análisis dinámico paso a paso de las estructuras. A partir de este análisis se obtienen las máximas distorsiones de entrepiso. Se determinan las medianas $(\hat{D})$ de dichas distorsiones y se ajustan los parámetros $a$ y $b$ de la ecuación 4. Además se evalúa la desviación estándar $\left(\sigma_{\ln D}\right)$ para diferentes niveles de intensidad sísmica. Con la ec. 9 se calcula el número mínimo de registros (n), para diferentes periodos de retorno $T_{R .}$.

Se obtiene la curva de peligro de demanda estructural $v_{D}(d)$ usando el método simplificado de análisis (ec. 5). A partir de esta se calcula la probabilidad de excedencia $P$ en un intervalo de tiempo dado $t$. Aquí se supone que el proceso es de Poisson, por lo que dicha probabilidad se expresa como sigue:

$$
P=1-\exp \left[-t v_{D}(d)\right]
$$

\section{MARCOS ESTRUCTURALES}

Se analizan tres marcos planos de concreto reforzado, que corresponden a edificios regulares con planta cuadrada. Un marco es de cinco niveles, otro de diez y el tercero de veinte niveles. Todos tienen tres crujías. Los dos primeros tienen un periodo fundamental $\left(T_{o}\right)$ igual a $1 \mathrm{~s}$, y el tercero tiene un periodo de $2.5 \mathrm{~s}$. Los edificios se ubican en la zona de terreno blando de la Ciudad de México, cercana al sitio SCT. En la fig. 2 se muestran las elevaciones de los marcos. 

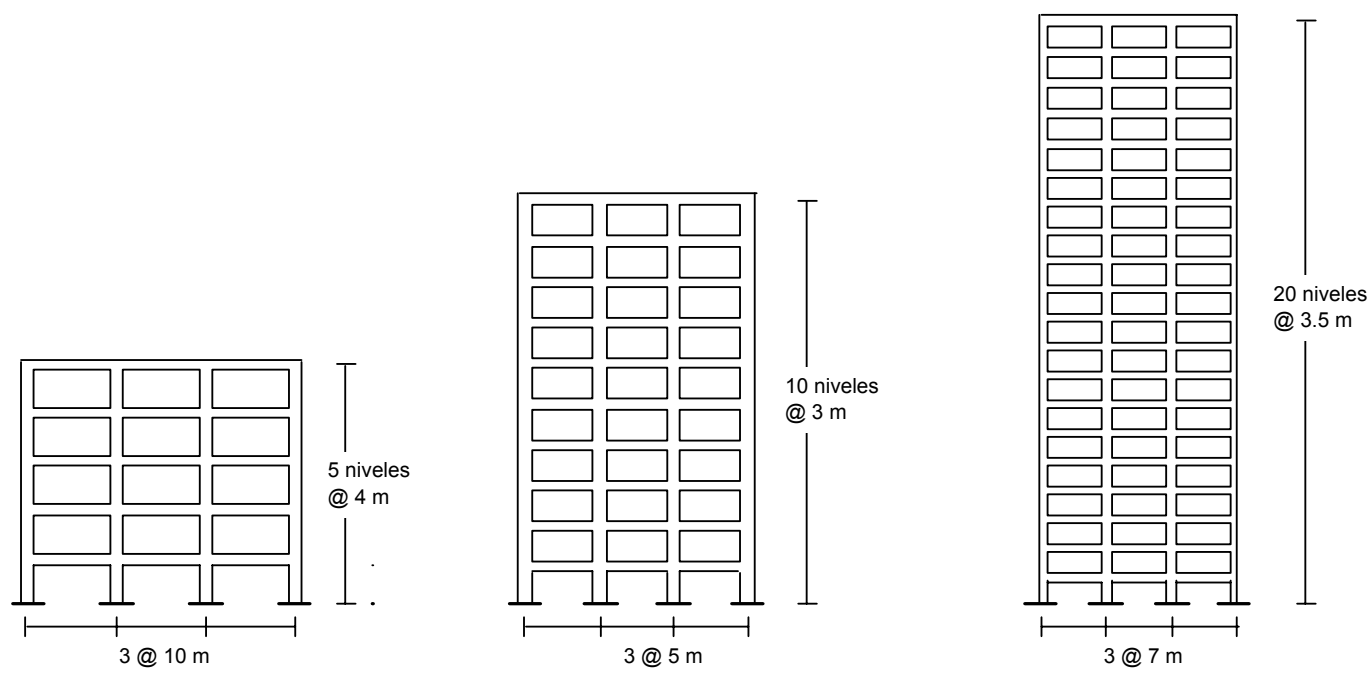

Figura 2. Elevación de los marcos

En lo que sigue se hace el análisis de confiabilidad de los tres marcos antes mencionados. Para cada uno se obtiene su curva de peligro de demanda sísmica, su probabilidad de excedencia en 50 años, y se calcula el número mínimo de movimientos sísmicos con la ec. 9.

\section{EDIFICIO DE CINCO NIVELES}

El primer edificio que se estudia consiste de cinco niveles y tres crujías. Presenta una distribución simétrica en planta. Este fue diseñado por la empresa de ingeniería PROYECT (Granados, 2001) de acuerdo con lo que especifica el RCDF-2003, con un factor de comportamiento sísmico $Q=3$. El edificio tiene un periodo de vibrar fundamental $T_{\mathrm{o}}=1 \mathrm{~s}$.

A partir de un análisis estático no lineal se obtuvo que el coeficiente sísmico de fluencia $\left(C_{\mathrm{s}}\right)$ del marco es igual a 0.31 . De análisis dinámicos paso a paso se encontró que la máxima distorsión se presenta generalmente en el segundo entrepiso.

En lo que sigue, se obtiene la confiabilidad estructural del marco en estudio usando tres criterios de escalamiento de acelerogramas. El primero considera $\mu=1, \xi=5 \%$; el segundo $\mu=$ $1, \xi=20 \%$, y el último $\mu=3, \xi=5 \%$. En los tres casos se considera que el periodo fundamental de la estructura es $T_{0}=1 \mathrm{~s}$.

Para realizar el análisis se supone conocida la curva de peligro sísmico asociada a los valores: $\mu=1, \xi=5 \%$ y $T_{\mathrm{o}}=1 \mathrm{~s}$ (fig. 3 ). A partir de dicha curva se obtuvieron otras curvas de peligro sísmico correspondientes a otros valores de $\mu$ y $\xi$. Para ello se usó el método numérico propuesto por Esteva (1976). Las curvas de peligro sísmico correspondientes a $\mu=1, \xi=20 \%$ y $T_{\mathrm{o}}=1 \mathrm{~s}$, y a $\mu=3, \xi=5 \%$ y $T_{\mathrm{o}}=1 \mathrm{~s}$, se presentan también en la fig. 3 . 




Figura 3. Curvas de peligro sísmico correspondientes a distintas demandas de ductilidad $(\mu)$, y a diferentes valores de amortiguamiento crítico $(\xi)$. Sitio SCT, $\mathrm{T}_{\mathrm{o}}=1 \mathrm{~s}$

\section{Número mínimo de registros requeridos para el análisis del edificio de cinco niveles}

En las figs. 4 a y b se muestran las medianas $\hat{D}$ y las desviaciones estándar $\sigma_{\ln D}$ de las máximas distorsiones de entrepiso demandadas, en función de distintos periodos de retorno $T_{R}$. Los resultados corresponden a los tres criterios de escalamiento mencionados antes. Los detalles del análisis se presentan en Chan (2003).

En la fig. 4a se observa que los valores de las medianas de las máximas distorsiones de entrepiso son similares para los movimientos con periodos de retorno pequeños $\left(T_{R}\right)$, y se separan ligeramente cuando los periodos de retorno se incrementan.

Por otro lado, las desviaciones estándar $\sigma_{\ln D}$ (fig. 4b) tienden a aumentar cuando crece el periodo de retorno. Esto se debe a que para periodos de retorno grandes las intensidades sísmicas son mayores, y la respuesta estructural presenta un comportamiento no lineal. En este caso la estructura responde con un periodo de vibración "equivalente" mayor que el original $\left(T_{o}=1 \mathrm{~s}\right)$. En la fig. 1 se puede observar que para periodos mayores que el original $\left(T_{o}=1 \mathrm{~s}\right)$ la dispersión de los movimientos es mayor.

En la tabla 2 se muestra el número mínimo de registros sísmicos requeridos, usando los tres criterios de escalamiento antes mencionados, para distintos periodos de retorno $\left(T_{R}\right)$. Los valores de la tabla 2 se obtuvieron sustituyendo valores en la ec. 9. 




a) Medianas, $\hat{D}$

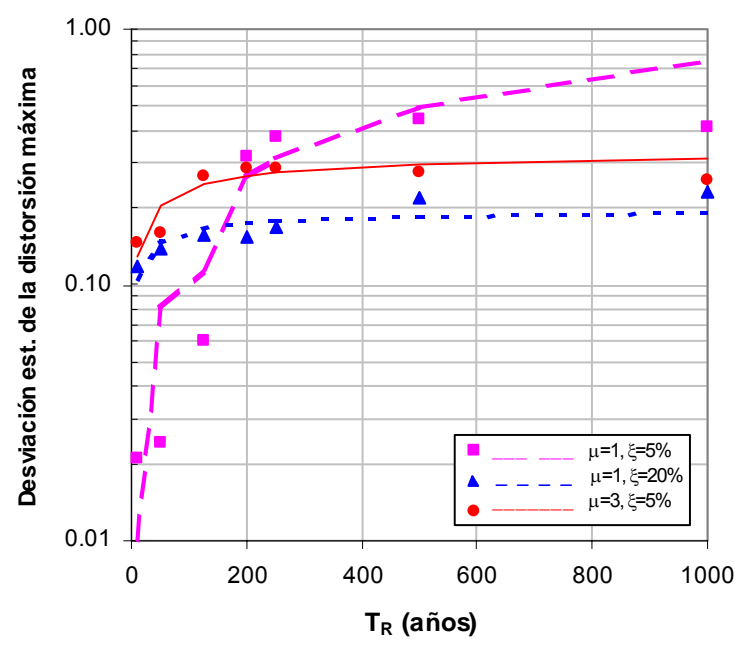

b) Desviaciones estándar, $\sigma_{\ln D}$

Figura 4. Estadísticos de las distorsiones máximas de entrepiso correspondientes a movimientos asociados a diferentes periodos de retorno. Marco de cinco niveles.

En la tabla 2 aparecen valores de números $n$ que no son enteros. Para que estos lo fueran debería aceptarse un error $e$ diferente a $10 \%$, o bien un valor de $K_{\alpha / 2}$ diferente a la unidad (Apéndice B). Para fines prácticos, más adelante se redondearán los números $n$ de la tabla 2 al valor próximo entero, en el entendido que en error que se acepta no es estrictamente de $10 \%$, sino cercano a este.

Tabla 2. Número de registros necesarios para el edificio de cinco niveles, usando tres criterios de escalamiento diferentes

\begin{tabular}{|c|c|c|c|}
\hline $\boldsymbol{T}_{\mathbf{R}}$ (años) & \multicolumn{3}{|c|}{ Número de registros $(\boldsymbol{n})$} \\
\hline & $\boldsymbol{\mu}=\mathbf{1 ,} \boldsymbol{\xi}=\mathbf{5 \%}, \boldsymbol{T}_{\mathbf{0}}=\mathbf{1} \mathbf{s}$ & $\boldsymbol{\mu}=\mathbf{1}, \boldsymbol{\xi}=\mathbf{2 0} \% \boldsymbol{T}_{\mathbf{0}}=\mathbf{1} \mathbf{s}$ & $\boldsymbol{\mu}=\mathbf{3 ,} \boldsymbol{\xi}=\mathbf{5 \%}, \boldsymbol{T}_{\mathbf{0}}=\mathbf{1 s}$ \\
\hline $\mathbf{1 0}$ & $\mathbf{0 . 0 1}$ & 1.13 & 1.68 \\
\hline $\mathbf{5 0}$ & $\mathbf{0 . 6 7}$ & 2.13 & 4.24 \\
\hline $\mathbf{1 2 5}$ & $\mathbf{1 . 3 0}$ & 2.74 & 6.15 \\
\hline 200 & 7.18 & $\mathbf{3 . 0 2}$ & 7.09 \\
\hline 250 & 9.84 & $\mathbf{3 . 1 3}$ & 7.50 \\
\hline 500 & 24.12 & $\mathbf{3 . 4 4}$ & 8.68 \\
\hline 1000 & 56.62 & $\mathbf{3 . 6 7}$ & 9.70 \\
\hline
\end{tabular}

Nótese que para periodos de retorno $\left(T_{R}\right)$ menores que 125 años el criterio de escalamiento que da lugar a un menor número de sismos es el de la segunda columna de la tabla 2 $\left(\mu=1, \xi=5 \%, T_{0}=1 \mathrm{~s}\right)$. Los números se indican con letra "negrita". Para periodos de retorno mayores que 125 años, el criterio que da lugar a un menor número de registros sísmicos es el de la tercera columna $\left(\mu=1, \xi=20 \%, T_{0}=1 \mathrm{~s}\right)$. Esto se debe a que ante sismos con intensidades altas la respuesta de la estructura tiene un comportamiento que es altamente no lineal. 
Las curvas de peligro de la demanda estructural (obtenidas con la ec. 5), y de probabilidad de excedencia de una distorsión dada (ec. 10), correspondiente a un intervalo de tiempo $t=50$ años, se presentan en las figs. $5 \mathrm{a}$ y $\mathrm{b}$.

Si se comparan las figs. 4a y 5a se observa que cuando las curvas de las fig. 4a se separan, también lo hacen las de la fig. 5a; es decir, que existe una marcada influencia de la mediana de la respuesta máxima en los valores de la confiabilidad estructural.

En las figs. 5a y b se puede ver que la confiabilidad estructural obtenida usando los tres criterios de escalamiento de acelerogramas es muy similar entre sí. Esto hace ver que el valor de la confiabilidad está implícito en la estructura, independientemente del criterio de escalamiento que se use. Las pequeñas diferencias que aparecen entre una y otra curva de las figs. $5 \mathrm{a}$ y $\mathrm{b}$ se deben a errores de aproximación en los ajustes de los parámetros de las ecuaciones 3 y 4 correspondientes a intensidades altas.

Lo anterior hace ver que los criterios de escalamiento no afectan el valor de la confiabilidad estructural, pero sí el del número mínimo de acelerogramas $n$.

La fig. 5 muestra que la probabilidad de que la distorsión máxima de entrepiso $(D)$ exceda el valor de 0.012 en 50 años es de aproximadamente $30 \%$, mientras que la probabilidad de que $D$ exceda el valor 0.004 es de $85 \%$ aproximadamente.

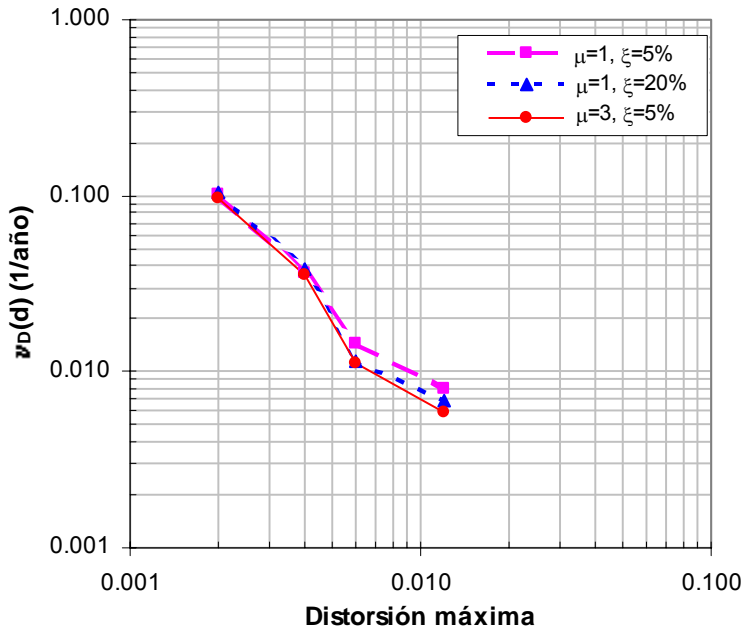

a) Tasa anual de excedencia de la distorsión máxima

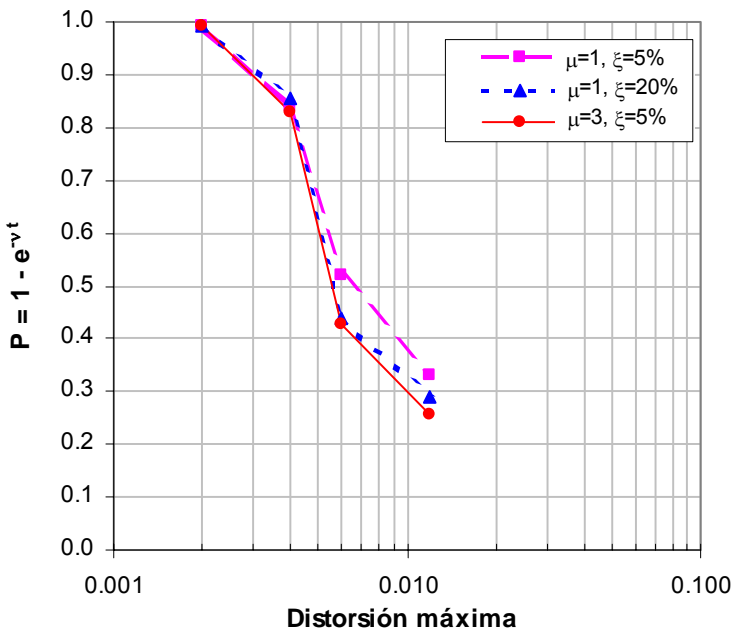

b) Probabilidad de excedencia de distorsión máxima en 50 años

Figura 5. Curvas de confiabilidad para el edificio de cinco niveles, correspondientes a tres criterios de escalamiento. 


\section{EDIFICIO DE DIEZ NIVELES}

El segundo edificio que se analiza en este estudio también tiene un periodo fundamental $T_{o}=1 \mathrm{~s}$. Se trata de un edificio de concreto reforzado de diez niveles con una altura de $3 \mathrm{~m}$ cada uno, y tres crujías de $5 \mathrm{~m}$; distancias medidas de eje a eje (fig. 2b). Al igual que el edificio de cinco niveles, tiene una distribución simétrica en planta, y un periodo de vibrar fundamental $T_{o}=1 \mathrm{~s}$ (Montiel, 2001).

El coeficiente sísmico de fluencia $\left(C_{\mathrm{s}}\right)$ se obtuvo mediante un análisis no lineal estático. Este es igual a 0.13 . Nótese que este valor es menor que lo que resiste el marco de cinco niveles, por lo que se espera que el de diez niveles tenga mayor comportamiento no lineal ante intensidades comparables y por lo tanto, la rigidez se degrade más, y el periodo "equivalente" se alargue más con respecto al edificio de cinco niveles.

De una serie de análisis con todos los sismos y para distintos periodos de retorno, se encontró que en la mayoría de los casos, la máxima distorsión de entrepiso en el marco de diez niveles se presenta en el segundo ó en el tercer entrepiso, dependiendo del nivel de demanda sísmica y del sismo que lo excita.

Para este marco se estudió la influencia de dos de los criterios de escalamiento mencionados antes. Estos corresponden a las siguientes características: $\mu=1, \xi=5 \%, T_{\mathrm{o}}=1 \mathrm{~s}, \mathrm{y}$ $\mu=1, \xi=20 \%, T_{\mathrm{o}}=1 \mathrm{~s}$.

Las curvas de peligro sísmico para $\mu=1, \xi=5 \%$ y $\mu=1, \xi=20 \%$ son las mismas que las del marco de cinco niveles (fig. 3), ya que el periodo fundamental de vibrar es el mismo. Como las curvas de peligro sísmico son las mismas, los factores de escala que afectan a los acelerogramas también son iguales.

En las figs. 6a y b se muestran las medianas y las desviaciones estándar de los logaritmos de las distorsiones máximas de entrepiso demandadas, correspondientes al edificio de diez niveles, correspondientes a los criterios de escalamiento mencionados antes. Se observa también que los valores de las medianas son muy semejantes, y que las desviaciones estándar son mayores cuando se usa el criterio de escalamiento correspondiente a $\mu=1, \xi=5 \%$ y $T_{0}=1 \mathrm{~s}$, que cuando se usa el correspondiente a $\mu=1, \xi=20 \%$ y $T_{0}=1 \mathrm{~s}$.

La curva de peligro de demanda y la de probabilidad de excedencia para un periodo de 50 años se muestran en las figs. $7 \mathrm{a}$ y b, respectivamente. En estas también se refleja que para una misma estructura y un mismo conjunto de sismos, la tasa anual de excedencia de la respuesta no depende del criterio de escalamiento que se utilice.

En este caso la probabilidad de que $D$ se exceda en 50 años del valor 0.012 es de $32 \%$ aproximadamente, mientras que la probabilidad de que $D$ sea mayor que 0.004 se aproxima a $80 \%$. 


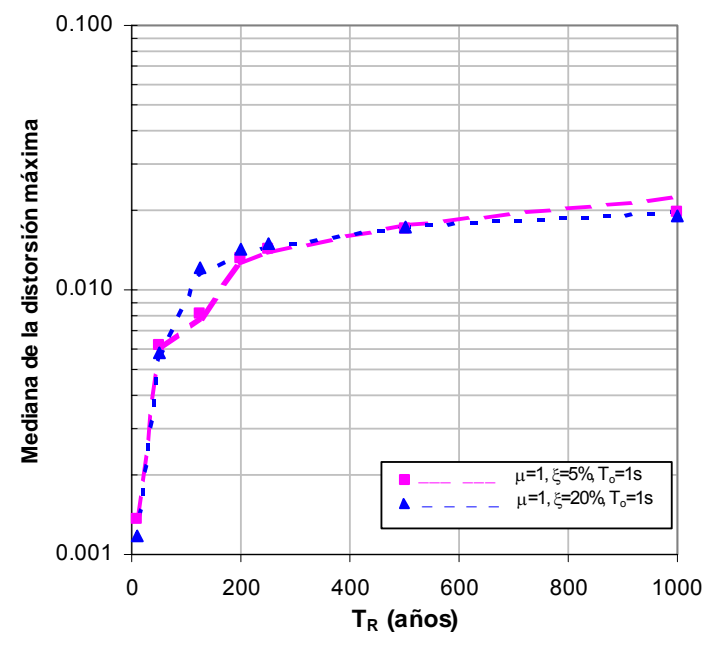

a) Medianas, $\hat{D}$

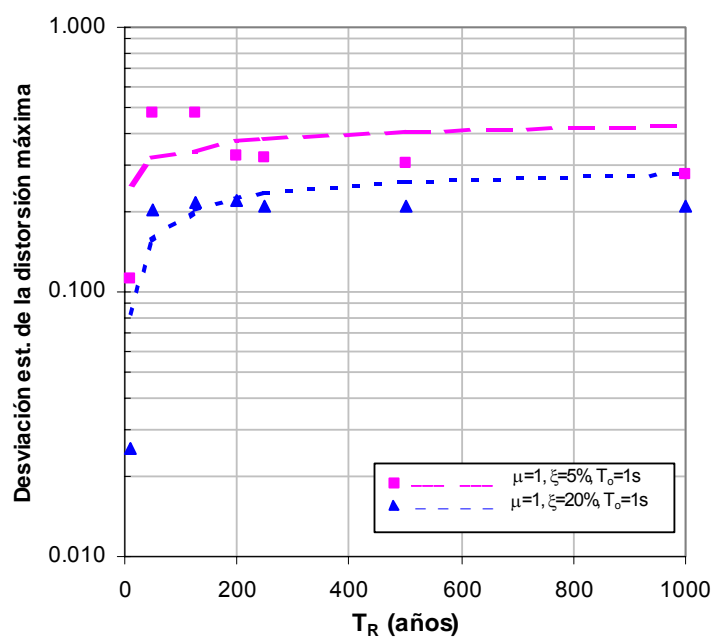

b) Desviaciones estándar, $\sigma_{\ln D}$

Figura 6. Estadísticos de las distorsiones máximas demandadas. Edificio de diez niveles

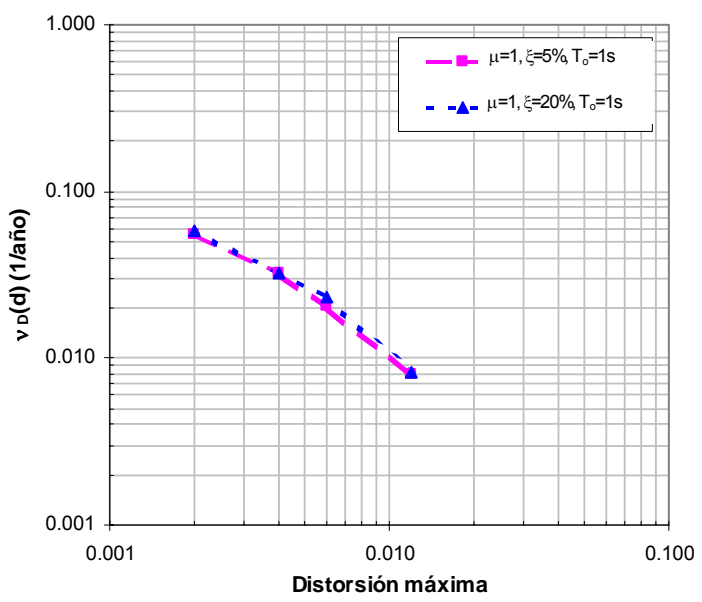

a) Tasa anual de excedencia de la distorsión

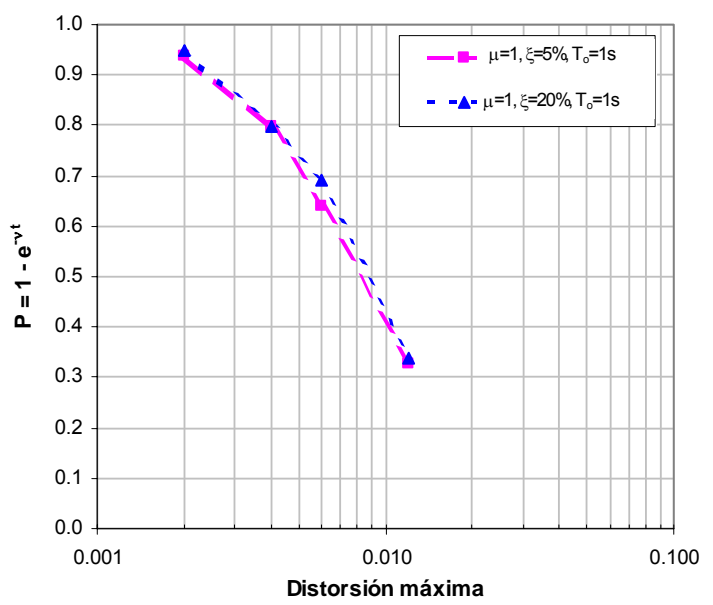

b) Probabilidad de excedencia de la distorsión máxima en 50 años

Figura 7. Curvas de confiabilidad para el edificio de diez niveles.

\section{Número mínimo de registros requeridos para el análisis del edificio de diez niveles}

Para el análisis del edificio de diez niveles se utilizó una metodología similar a la empleada para analizar el marco de cinco niveles. En la tabla 3 se presenta el número de registros necesarios obtenidos con la ec. 9, según dos criterios de escalamiento: $\left(\mu=1, \xi=5 \%, T_{\mathrm{o}}=1 \mathrm{~s}\right.$, y $\mu=1, \xi=$ $\left.20 \%, T_{\mathrm{o}}=1 \mathrm{~s}\right)$. Si se compara esta tabla con la tabla 3 se deduce que para el edificio de diez 
niveles se requiere mayor número $(n)$ de registros para obtener la misma banda de confianza y el mismo error $e$, que para el marco de cinco niveles. Esto se debe a que el edificio de diez niveles presentó mayor comportamiento no lineal, y mayor dispersión en su respuesta estructural, para movimientos con periodos de recurrencia similares (esto obedece a que el coeficiente sísmico de fluencia es menor que el del marco de cinco niveles).

Tabla 3. Número mínimo de registros (n) para el edificio de diez niveles

\begin{tabular}{|c|c|c|}
\hline $\mathbf{T}_{\mathbf{R}}$ (años) & $\boldsymbol{\mu}=\mathbf{1}, \boldsymbol{\xi}=\mathbf{5 \%}, \boldsymbol{T}_{\mathbf{0}}=\mathbf{1 s}$ & $\boldsymbol{\mu}=\mathbf{1 ,} \boldsymbol{\xi}=\mathbf{2 0 \%} \boldsymbol{T}_{\mathbf{0}}=\mathbf{1 s}$ \\
\hline $\mathbf{1 0}$ & 1.21 & $\mathbf{0 . 6 6}$ \\
\hline $\mathbf{5 0}$ & 10.11 & $\mathbf{2 . 2 7}$ \\
\hline $\mathbf{1 2 5}$ & 10.85 & $\mathbf{3 . 7 3}$ \\
\hline 200 & 12.98 & $\mathbf{4 . 5 1}$ \\
\hline 250 & 13.41 & $\mathbf{4 . 8 6}$ \\
\hline 500 & 14.73 & $\mathbf{5 . 8 3}$ \\
\hline 1000 & 16.11 & $\mathbf{6 . 6 3}$ \\
\hline
\end{tabular}

La tabla 3 muestra (con letras "negritas") que, para cualquier nivel de intensidad sísmica, el criterio de escalamiento de la tercera columna $\left(\mu=1, \xi=20 \%\right.$ y $\left.\mathrm{T}_{\mathrm{o}}=1 \mathrm{~s}\right)$ requiere menor número de registros que cuando se usa el criterio de la segunda columna $\left(\mu=1, \xi=5 \%\right.$ y $\mathrm{T}_{\mathrm{o}}=$ $1 \mathrm{~s})$.

\section{EDIFICIO DE VEINTE NIVELES}

El tercer edificio que se analiza tiene veinte niveles. Consta de tres crujías de $7 \mathrm{~m}$ y la altura de cada nivel es de $3.5 \mathrm{~m}$, medidos de eje a eje. Al igual que los edificios anteriormente analizados, tiene una distribución simétrica en planta. Para efectuar el análisis estructural se modeló solamente un marco central. El periodo de la estructura es de $2.5 \mathrm{~s}$. El coeficiente sísmico obtenido a partir de un análisis no lineal estático resultó igual a 0.12 . A diferencia de los edificios anteriores, este se diseñó de acuerdo con el Reglamento de Construcciones del Distrito Federal de 1976 (RCDF-1976), y no cumple con las distorsiones máximas de entrepiso especificadas en el RCDF-2004.

Tabla 4. Propiedades dinámicas del marco de veinte niveles

\begin{tabular}{|c|c|c|c|}
\hline Modo & $\boldsymbol{f}(\mathbf{H z})$ & $\boldsymbol{T}(\mathbf{s})$ & Masa modal $(\mathbf{\%})$ \\
\hline 1 & 0.39 & 2.510 & 0.751 \\
\hline 2 & 1.18 & 0.846 & 0.121 \\
\hline 3 & 2.08 & 0.480 & 0.041 \\
\hline 4 & 3.02 & 0.330 & 0.022 \\
\hline 5 & 4.06 & 0.246 & 0.015 \\
\hline
\end{tabular}


En la tabla 4 se presentan las propiedades dinámicas del marco. Estas corresponden a los primeros cinco modos de vibrar, que son los que tienen mayor influencia en la respuesta estructural. De las propiedades dinámicas obtenidas se puede deducir que el porcentaje de masa modal en el segundo modo es relativamente importante en la respuesta del edificio.

Las distorsiones máximas se presentan principalmente en el noveno entrepiso cuando la estructura se somete a sismos con intensidades bajas; sin embargo, a medida que las intensidades aumentan, la distorsión máxima se presenta generalmente en el octavo entrepiso.

Para este edificio se analiza la confiabilidad estructural usando tres criterios de escalamiento. El primero considera $\mu=1, \xi=5 \%, T_{\mathrm{o}}=2.5 \mathrm{~s}$, el segundo considera $\mu=1, \xi=$ $20 \%, T_{\mathrm{o}}=2.5 \mathrm{~s}$, y el tercero es el criterio de escalamiento $(d)$ mencionado en la sección "Criterios de escalamiento de acelerogramas" de este artículo. En este último criterio se asignan factores de peso a los modos de vibrar más importantes, por lo que se le llama criterio de pesos modales (Shome y Cornell, 1999; Chan, 2003).

En el criterio de pesos modales se dan pesos a los modos de vibrar más importantes para definir los valores de las seudoaceleraciones espectrales $(\mathrm{Sa} / \mathrm{g})$, en función de los porcentajes de masa modal efectiva del edificio (tabla 4). En este ejemplo al primer modo se le asigna un porcentaje igual a $80 \%$, al segundo $15 \%$, y al tercero $5 \%$. La suma de porcentajes resulta el cien por ciento. De esta manera, las ordenadas espectrales en el periodo correspondiente a cada modo se multiplican por los porcentajes que se les ha asignado. La intensidad con la que se determina el factor de escala, es la suma de las seudoaceleraciones espectrales entre la gravedad por su porcentaje de peso. Esta intensidad se denota $(\mathrm{Sa} / \mathrm{g})_{\text {modal }}$. Con estas intensidades y las encontradas de la curva de peligro sísmico $\left(\mu=1, \xi=20 \%\right.$ y $\left.T_{\mathrm{o}}=2.5 \mathrm{~s}\right)$ se calculan los factores de escala correspondientes a este criterio (los detalles del análisis se presentan en Chan, 2003).

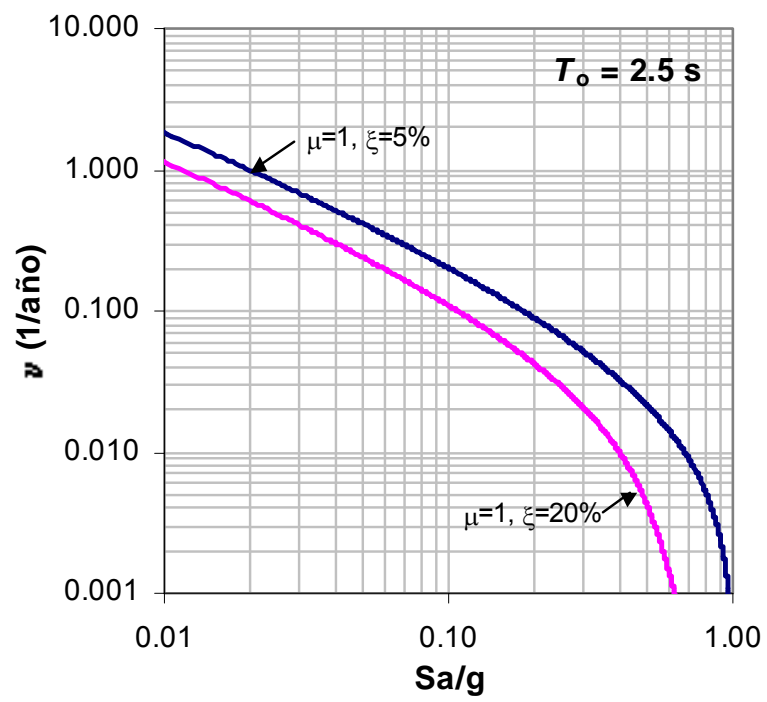

Figura 8. Curvas de peligro sísmico $T_{0}=2.5 \mathrm{~s}$. Sitio $\mathrm{SCT}$. 
Para el criterio de escalamiento $\mu=1, \xi=20 \%$ y pesos modales, se usó la misma curva de peligro sísmico correspondiente a $\mu=1, \xi=20 \%$ y $T_{0}=2.5 \mathrm{~s}$ (fig. 8). En este caso se usó la curva de peligro sísmico para el periodo de vibración correspondiente al primer modo de vibrar $\left(T_{\mathrm{o}}=2.5 \mathrm{~s}\right)$, ya que la participación de este modo es mucho mayor, comparada con la del segundo y la del tercer modo.

\section{Número mínimo de registros requeridos para el análisis del edificio de veinte niveles}

Las medianas y desviaciones estándar del logaritmo de las distorsiones máximas de entrepiso que se obtienen al usar los tres criterios mencionados antes se presentan en las figs. 9a y b.

La fig. 9a muestra que las medianas de las distorsiones máximas de entrepiso son muy similares para todos los periodos de retorno cuando se emplea cualquiera de los tres criterios de escalamiento. En la fig. 9b se puede apreciar la influencia de los tres diferentes criterios de escalamiento en las desviaciones estándar de las distorsiones máximas de entrepiso.

En la fig. $9 \mathrm{~b}$ se puede observar que el criterio de escalamiento que utiliza $\mu=1, \xi=5 \%$ y $T_{\mathrm{o}}=2.5 \mathrm{~s}$ da lugar a desviaciones estándar del logaritmo de la respuesta más pequeñas que las de los otros criterios; por lo tanto, se obtiene un menor número de registros (tabla 5). Le sigue el criterio $\mu=1, \xi=20 \%$ y $T_{0}=2.5 \mathrm{~s}$, y por último el criterio de pesos modales.

De aquí se deduce que el criterio de escalamiento más adecuado para el edificio de veinte niveles es el que considera $\mu=1, \xi=5 \%, \mathrm{~T}_{\mathrm{o}}=1 \mathrm{~s}$. Este criterio tiene la ventaja de que no se tienen que construir curvas de peligro sísmico para amortiguamientos diferentes a cinco por ciento.



a) Medianas, $\hat{D}$

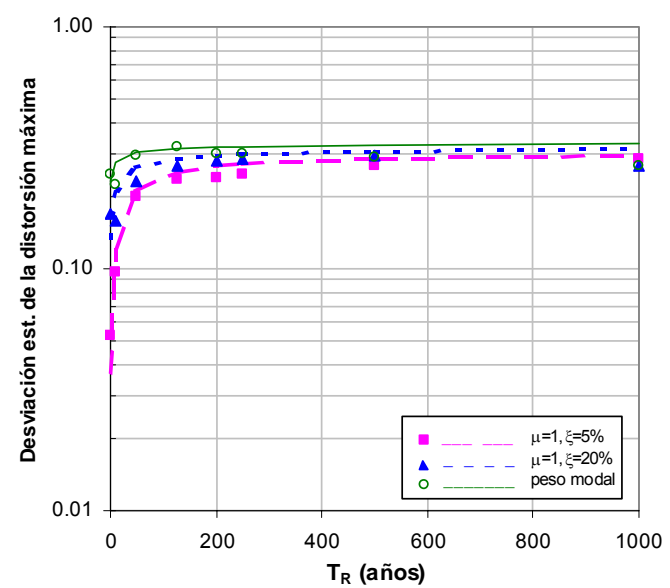

b) Desviaciones estándar, $\sigma_{\ln D}$

Figura 9. Estadísticos de las distorsiones máximas demandadas correspondientes al edificio de veinte niveles $\left(T_{0}=2.5 \mathrm{~s}\right)$. 
La similitud en el valor de las medianas que se presentan en la fig. 9a se refleja en la confiabilidad estructural, ya que tanto las curvas de tasa anual de excedencia de las distorsiones máximas, como la curva de probabilidad de excedencia de la distorsión en 50 años, son similares (figs. 10a y b). Con esto se verifica la estrecha relación que existe entre las medianas de la respuesta máxima estructural y la confiabilidad estructural, como se mencionó anteriormente.

Tabla 5. Número de registros necesarios para el marco de veinte niveles

\begin{tabular}{|c|c|c|c|}
\hline \multirow{2}{*}{$\boldsymbol{T}_{\mathbf{R}}(\mathbf{a n ̃ o s})$} & \multicolumn{3}{|c|}{ Número de registros (n) } \\
\cline { 2 - 4 } & $\boldsymbol{\mu}=\mathbf{1 ,} \boldsymbol{\xi}=\mathbf{5 \%}, \boldsymbol{T}_{\mathbf{0}}=\mathbf{2 . 5 s}$ & $\boldsymbol{\mu}=\mathbf{1 ,} \boldsymbol{\xi}=\mathbf{2 0} \boldsymbol{\mathbf { o }}, \boldsymbol{T}_{\mathbf{0}}=\mathbf{2 . 5} \mathbf{s}$ & Pesos modales \\
\hline $\mathbf{1}$ & $\mathbf{0 . 1 3}$ & 1.79 & 5.19 \\
\hline $\mathbf{1 0}$ & $\mathbf{1 . 2 8}$ & 4.17 & 7.12 \\
\hline $\mathbf{5 0}$ & $\mathbf{3 . 7 3}$ & 6.18 & 8.25 \\
\hline $\mathbf{1 2 5}$ & $\mathbf{5 . 3 0}$ & 7.04 & 8.66 \\
\hline 200 & $\mathbf{5 . 9 5}$ & 7.37 & 8.81 \\
\hline 250 & $\mathbf{6 . 2 1}$ & 7.50 & 8.87 \\
\hline 500 & $\mathbf{6 . 8 1}$ & 7.85 & 9.02 \\
\hline 1000 & $\mathbf{7 . 1 5}$ & 8.12 & 9.14 \\
\hline
\end{tabular}

En las figs. 10a y b se observa que las curvas de confiabilidad obtenidas con tres diferentes criterios de escalamiento son similares, como era de esperarse.

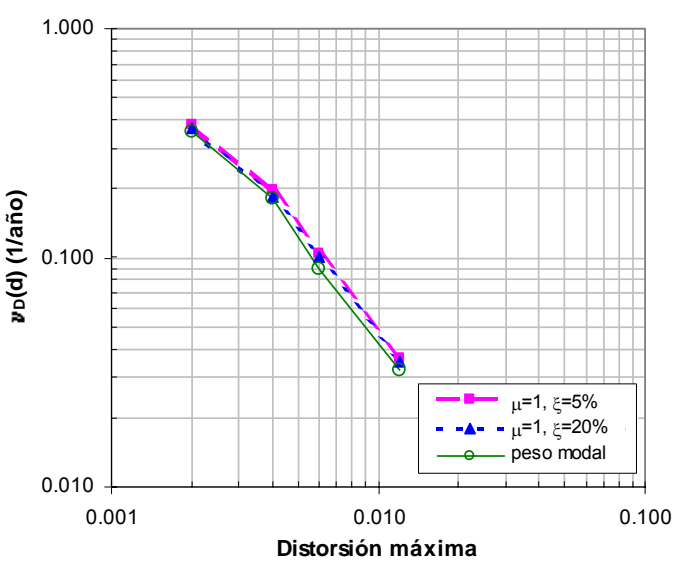

a) Tasa anual de excedencia

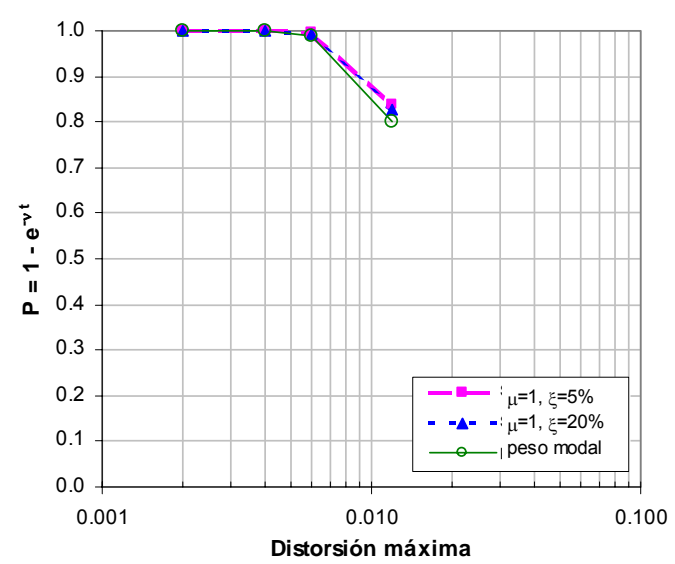

b) Probabilidad de excedencia de la distorsión máxima en 50 años

Figura 10. Curvas de confiabilidad para el edificio de veinte niveles. 
En este caso la probabilidad de que $D$ se exceda del valor 0.012 en 50 años es de aproximadamente $82 \%$, mientras que la probabilidad de que $D$ se exceda de 0.004 es de $100 \%$. Nótese que estas probabilidades de excedencia son mucho mayores a las de los marcos de cinco y de diez niveles, debido a que este marco se diseñó con el RCDF-76 y los otros dos con el RCDF2004.

\section{ANÁLISIS DE RESULTADOS Y CONCLUSIONES}

Las conclusiones que se mencionan en lo que sigue se aplican a movimientos de banda angosta, como los registrados en la estación SCT ubicada en terreno blando de la ciudad de México.

\section{Sobre la confiabilidad de las estructuras}

En este estudio se mencionó que las curvas de peligro de demanda estructural dependen en gran medida de las medianas de las distorsiones máximas. Para los casos que se analizan aquí, la mediana de la respuesta (en este caso de la máxima distorsión de entrepiso) tiene una gran influencia en los valores de la curva de peligro de demanda y en la probabilidad de excedencia asociada a cierto intervalo de tiempo. Debido a esto, se recomienda verificar que la aproximación en el ajuste de las medianas de las distorsiones máximas sea riguroso, ya que de no ser así se pueden obtener estimaciones erróneas de la confiabilidad estructural.

El estudio de confiabilidad estructural de los tres marcos analizados indica que los edificios con periodo fundamental de vibrar $T_{o}=1 \mathrm{~s}$ presentan una confiabilidad muy diferente al edificio con $T_{o}=2.5 \mathrm{~s}$. Esto se debe en parte a que este último edificio fue diseñado con el RCDF1976, y no con el RCDF-2004. Por otro lado, aún cuando se hubiese diseñado con este último reglamento, es muy probable que las confiabilidades de los edificios de todas maneras serían muy diferentes, pues el RCDF (como la mayoría de los reglamentos del mundo) no establece la condición de igual confiabilidad para edificios con diferentes periodos de vibración.

En este estudio se proporciona información útil sobre las probabilidades de falla en cierto intervalo de tiempo de edificios diseñados de acuerdo con el RCDF. Las figs. 5, 7 correspondientes a las estructuras de cinco y diez niveles $\left(T_{o}=1 \mathrm{~s}\right)$ dan idea sobre la confiabilidad implícita en diseños realizados de acuerdo con el RCDF-2004. Por otro lado, la fig. 10 proporciona información sobre la confiabilidad de edificios con periodo $T_{o}=2.5 \mathrm{~s}$, localizados en un sitio cercano a la estación SCT, diseñados con el RCDF-1976.

\section{Sobre los criterios de escalamiento}

Cuando el marco de cinco niveles $\left(T_{o}=1 \mathrm{~s}\right)$ se excita con sismos escalados a periodos de recurrencia menores que 125 años, desarrolla un comportamiento casi lineal. Debido a esto, resultó más conveniente utilizar el criterio de escalamiento $\mu=1, \xi=5 \%$ y $\mathrm{T}_{\mathrm{o}}=1 \mathrm{~s}$. Esto se deduce a partir de que el número de registros $n$ es menor que para los otros criterios analizados (tabla 2). 
Para el marco de diez niveles $\left(T_{o}=1 \mathrm{~s}\right)$ resultó más conveniente utilizar el criterio de escalamiento que considera $\mu=1, \xi=20 \%$ y $T_{o}=1$ s (tabla 3 ). El inconveniente de utilizar el criterio $\mu=1, \xi=20 \%$ y $T_{o}$ es que las curvas de peligro sísmico generalmente se construyen para un valor de $\xi=5 \%$, por lo que se deberá contar con información suficiente para construir la curva de peligro sísmico correspondiente a $\xi=20 \%$.

Para el marco de veinte niveles $\left(T_{o}=2.5 \mathrm{~s}\right)$, resultó más conveniente utilizar el criterio de escalamiento $\mu=1, \xi=5 \%$ y $T_{o}$ (tabla 5 ).

\section{Sobre el número de registros necesarios para tener una banda de confianza dada}

De las tablas 2, 3 y 5 se deduce que, cuando se estudian estructuras sujetas a sismos poco intensos ( $T_{\mathrm{R}}=10$ años o menos), basta un solo movimiento típico del sitio en estudio para estimar que la mediana de las distorsiones máximas de entrepiso cae dentro de una banda de confianza $K_{\alpha / 2}$ unitaria.

Por otro lado, cuando se estudian las estructuras ante sismos con periodo de recurrencia $T_{R}=125$ años, el número mínimo de registros necesarios varía entre uno y cinco, dependiendo del periodo de vibrar de la estructura y del coeficiente sísmico resistente ante cargas laterales $\left(C_{s}\right)$. Esto se indica en la tabla 6.

Se hace la observación de que el número de registros que recomienda la propuesta del Reglamento de Construcciones para el D.F., en sus Normas Técnicas Complementarias para Diseño por Sismo (NTCS-2004) es de "no menos de cuatro movimientos sísmicos". Este número resultó mayor o igual que los que se requirieron para revisar los estados límite de servicio $\left(T_{R}=\right.$ 10 años) y de falla ( $T_{R}=125$ años) de los edificios de cinco y de diez niveles analizados en este trabajo (tabla 6); pero resultó menor que los que se requirieron para analizar el edificio de veinte niveles. Esto puede deberse a que este último se diseñó con la versión NTCS de 1976, y no con la última versión de NTCS.

Tabla 6. Resumen del número mínimo de registros sísmicos. $T_{R}=125$ años.

\begin{tabular}{|l|c|c|c|}
\hline & \multicolumn{3}{|c|}{ Número mínimo de registros (n) } \\
\hline & \multicolumn{2}{|c|}{ Edificios con $\mathbf{T}_{\mathbf{0}}=\mathbf{1 s}$} & Edificio con $\mathbf{T}_{\mathbf{o}}=\mathbf{2 . 5 s}$ \\
\hline \multirow{3}{*}{} & Cinco niveles & Diez niveles & Veinte niveles \\
& $\mathbf{C}_{\mathbf{s}}=\mathbf{0 . 3 1}$ & $\mathbf{C}_{\mathbf{s}}=\mathbf{0 . 1 3}$ & $\mathbf{C}_{\mathbf{s}}=\mathbf{0 . 1 2}$ \\
\hline$T_{R}=125$ años & $1.3 \triangleright 1$ & $3.7-4$ & $5.3 \triangleright 5$ \\
\hline
\end{tabular}

El presente estudio es útil porque se sistematizó el procedimiento de análisis para encontrar el valor de $n$ y de la confiabilidad estructural, y porque proporciona una idea general sobre los valores aceptables del número mínimo de acelerogramas $(n)$ que deben utilizarse para el análisis dinámico de las estructuras. 
Con el fin de proporcionar intervalos de valores del número de registros que deben recomendarse en un reglamento de construcciones se recomienda analizar edificios con distintas características estructurales, diferentes coeficientes sísmicos de diseño, y diferentes tipos de excitaciones sísmicas a las que se verán sometidas las estructuras.

El estudio deberá extenderse para definir el nivel de confianza y el error óptimo a partir de estudios de confiabilidad.

\section{AGRADECIMIENTOS}

Los autores agradecen a Raúl Granados Granados su valioso apoyo en el diseño estructural, a Mario Ordaz los comentarios críticos al manuscrito, y a Marco Antonio Torres Pérez-Negrón su apoyo y atinadas observaciones. Se agradece el patrocinio de la DGAPA-UNAM para el desarrollo de este proyecto.

\section{REFERENCIAS}

Ang, A y W Tang (1975), Probability concepts in engineering planning and design, John Wiley \& Sons.

Benjamin, J R y C A Cornell (1970), Probability, statistics and decisions for civil engineers, McGraw Hill.

Chan, S M (2003), "Influencia del escalamiento de movimientos sísmicos de banda estrecha en la evaluación de la confiabilidad de edificios", Tesis de Maestría, División de Estudios de Posgrado de la Facultad de Ingeniería, UNAM.

Cornell, C A (1996), "Calculating building seismic performance reliability basis for multilevel design norms", Memorias, $11^{\text {th }}$ World Conference on Earthquake Engineering, Acapulco, México, Artículo No 2122.

Esteva, L (1976), "Capítulo 6: Seismicity”, Seismic risk and engineering decisions, C. Lomnitz y E. Rosenblueth (editores), Elsevier, pp. 179-224.

Granados, R (2001), Comunicación personal.

Matsumura, K (1992), "On the intensity measure of strong motions related to structural failures", Memorias, $10^{\text {th }}$ World Conference on Earthquake Engineering, Madrid, España, Vol. 1, pp. 375380 .

Montiel, M A (2001), “Casos en que los disipadores de energía constituyen una mejor opción que los contravientos para reforzar edificios", Tesis de Maestría, División de Estudios de Posgrado de la Facultad de Ingeniería, UNAM.

Montiel, M A, S E Ruiz. y S Chan (2002), "Evaluación de la confiabilidad sísmica de construcciones usando un método simplificado", Memorias, VIII Jornadas Chilenas de Sismología e Ingeniería Antisísmica, Valparaíso, Chile.

Montiel, M A, S E Ruiz y M A Torres (2003), "Comparison of two alternative methods for 
estimating annual mean failure rates of building frames", Memorias, International Conference Risk, Vulnerability and Reliability in Construction, Argel, Argelia, pp. 524-534.

NTCS-2004 (2004), "Normas Técnicas Complementarias para Diseño por Sismo", Gaceta Oficial del Distrito Federal, octubre, México, D. F.

Shome, N, C A Cornell, P Bazzurro y J E Carballo (1998), "Earthquakes, records and nonlinear responses", Earthquake Spectra, Vol. 14, No. 3, pp. 469-500.

Shome N y C A Cornell (1999), "Probabilistic seismic demand analysis of nonlinear structures", Reliability of Marine Structures Program, Report No. RMS-35, Dept. of Civil Eng., Stanford University, Stanford, CA.

Villa, C y S E Ruiz (2001), "Influencia de la intensidad en las características de los movimientos registrados en el Valle de México", Informe elaborado para la Secretaría de Obras y Servicios del Gobierno del Distrito Federal, Convenio GDF-UNAM CT/23/01. México, D. F.

\section{APÉNDICE A: DEDUCCIÓN DE LA ECUACIÓN 2}

Se parte de que la función de densidad de probabilidad (fdp) de la distorsión máxima de entrepiso es lognormal (Shome y Cornell, 1999). Esto se expresa como:

$$
f_{D}(d)=\frac{1}{d \sqrt{2 \pi} \sigma_{\ln D}} \exp \left[-\frac{1}{2}\left(\frac{1}{\sigma_{\ln D}} \ln \left(\frac{d}{\hat{D}}\right)\right)^{2}\right]
$$

Su función de distribución acumulada se expresa como:

$$
F_{D}(d)=P(D \leq d)=P(\ln D \leq \ln d)
$$

Se define la variable estandarizada $\mathrm{u}$, con fdp normal, con media nula y desviación estándar unitaria, como sigue:

$$
u=\frac{\ln D-\ln \hat{D}}{\sigma_{\ln D}}
$$

Si se despeja $\ln D$ de esta última y se sustituye en la ec. A2, se obtiene: 
$F_{D}(d)=P\left(u \sigma_{\ln D}+\ln \hat{D} \leq \ln d\right)=P\left(u \leq\left[\frac{\ln (d / \hat{D})}{\sigma_{\ln D}}\right]\right)$

De aquí se deduce que:

$P(D \leq d)=P\left(u \leq\left[\frac{\ln (d / \hat{D})}{\sigma_{\ln D}}\right]\right) \quad F_{u}\left[\frac{\ln (d / \hat{D})}{\sigma_{\ln D}}\right]=\Phi(u)$

donde $\Phi(u)$ es la función de distribución acumulada normal estándar.

De lo anterior se deduce la ec. 2 del presente artículo:

$P(D \geq d)=1-\Phi\left(\frac{\ln (d / \hat{D}}{\sigma_{\ln D}}\right)$

\section{APÉNDICE B: DEDUCCIÓN DE LA ECUACIÓN 9}

El valor del estimador (o sea la distorsión máxima de entrepiso) se obtiene a partir de un número limitado de respuestas estructurales. Estas pueden considerarse muestras de un conjunto de variables aleatorias independientes $x_{1}, x_{2}, \ldots x_{n}$ cuyas fdp son iguales a las de las variables $X_{1}$, $X_{2}, \ldots X_{n}$ (Ang y Tang, 1975). En donde $X=\ln D$, o sea el logaritmo natural del valor de las distorsiones máximas de entrepiso.

El valor medio de la muestra está dada por:

$\dddot{X}=\frac{1}{n} \sum_{i=1}^{n} X_{i}$

$\mathrm{Su}$ valor esperado es:

$E(\dddot{X})=\frac{1}{n} \sum_{i=1}^{n} E\left(X_{i}\right)=\frac{1}{n} n \mu=\mu$

Es decir que el valor esperado de la media de la muestra es igual a la media de la población $(\mu)$, por lo que se dice que $\dddot{X}$ es un estimador no sesgado de $\mu$. 
La varianza de $\dddot{X}$ es igual a:

$$
\frac{1}{n^{2}}\left(n \sigma^{2}\right)=\frac{\sigma^{2}}{n} \quad \operatorname{Var} \dddot{X}=\frac{1}{n^{2}} \operatorname{Var}\left(\sum_{i=1}^{n} X_{i}\right)
$$

En resumen $X$ tiene media $\mu$, y desviación estándar $\sigma / \sqrt{n}$. Debido al Teorema del Límite Central, la fdp de $X$ es Gaussiana.

Enseguida se define la variable estándar $u$ :

$$
u=\frac{\dddot{X}-\mu}{\sigma / \sqrt{n}}
$$

La probabilidad de que $u$ se encuentre dentro de cierto intervalo $-K_{\alpha / 2}$ y $K_{\alpha / 2}$ es igual a:

$$
P\left(-K_{\alpha / 2} \leq \frac{\dddot{X}-\mu}{\sigma / \sqrt{n}} \leq K_{\alpha / 2}\right)=\Phi\left(K_{\alpha / 2}\right)-\Phi\left(-K_{\alpha / 2}\right)=1-\alpha
$$

De donde se obtiene lo siguiente:

$$
P\left(\dddot{X}-K_{\alpha / 2} \frac{\sigma}{\sqrt{n}} \leq \mu \leq \dddot{X}+K_{\alpha / 2} \frac{\sigma}{\sqrt{n}}\right)=1-\alpha
$$

Esto indica que hay a confianza igual a $1-\alpha$ de que el valor de $\mu$ se encuentre en el intervalo $\left(X-K_{\alpha / 2} \frac{\sigma}{\sqrt{n}}, X+K_{\alpha / 2} \frac{\sigma}{\sqrt{n}}\right)$. Es decir que el intervalo de confianza depende de la desviación estándar $\sigma$, del valor de $K_{\alpha / 2}, y$ del número de muestras $n$ (en este caso, del número de acelerogramas considerados para el análisis). El error de estimación ( $e$ ) que se comete al usar $\dddot{X}$ para estimar el valor de la media poblacional es el siguiente:

$e=K_{\alpha / 2} \sigma / \sqrt{n}$

Despejando se obtiene:

$$
n=K_{\alpha / 2}^{2} \frac{\sigma^{2}}{e^{2}}=K_{\alpha / 2}^{2} \frac{\sigma_{\ln D}^{2}}{e^{2}}
$$

